\title{
Association of the KLF14 rs4731702 SNP and Serum Lipid Levels in the Guangxi Mulao and Han Populations
}

\author{
Ping Huang, ${ }^{1}$ Rui-Xing Yin, ${ }^{1}$ Ke-Ke Huang, ${ }^{1}$ Xiao-Na Zeng, ${ }^{1}$ Tao Guo, ${ }^{1}$ \\ Quan-Zhen Lin, ${ }^{1}$ Jian Wu, ${ }^{1}$ Dong-Feng Wu, ${ }^{1}$ Hui Li, ${ }^{2}$ and Shang-Ling Pan ${ }^{3}$ \\ ${ }^{1}$ Department of Cardiology, Institute of Cardiovascular Diseases, The First Affiliated Hospital, Guangxi Medical University, \\ Nanning, Guangxi 530021, China \\ ${ }^{2}$ Clinical Laboratory of the Affiliated Cancer Hospital, Guangxi Medical University, Nanning, Guangxi 530021, China \\ ${ }^{3}$ Department of Pathophysiology, School of Premedical Sciences, Guangxi Medical University, Nanning, Guangxi 530021, China
}

Correspondence should be addressed to Rui-Xing Yin; yinruixing@sohu.com

Received 9 April 2013; Revised 17 August 2013; Accepted 26 August 2013

Academic Editor: Zhirong Sun

Copyright (C) 2013 Ping Huang et al. This is an open access article distributed under the Creative Commons Attribution License, which permits unrestricted use, distribution, and reproduction in any medium, provided the original work is properly cited.

\begin{abstract}
The objective of the present study was to detect the association of the rs4731702 single nucleotide polymorphism (SNP) and serum lipid levels in the Guangxi Mulao and Han populations. A total of 727 subjects of Mulao and 740 subjects of Han Chinese were included. Serum low-density lipoprotein cholesterol (LDL-C) and apolipoprotein (Apo) B levels were higher in Mulao than in Han $(P<0.05)$. The T allele carriers had higher serum LDL-C and ApoAI levels in Mulao, whereas they had lower high-density lipoprotein cholesterol (HDL-C) levels and ratio of ApoAI to ApoB in Han $(P<0.05)$ than the T allele noncarriers. Subgroup analyses showed that the T allele carriers had higher HDL-C, LDL-C, and ApoAI levels in Mulao males and lower ApoAI levels and ratio of ApoAI to ApoB in Han males than the T allele noncarriers. The subjects with TT genotype in Han females also had higher total cholesterol, LDL-C, ApoAI, and ApoB levels than the subjects with CT or CC genotype. Serum lipid parameters were also correlated with several environmental factors in both ethnic groups. The differences in the association of KLF14 rs4731702 SNP and serum lipid levels between the two ethnic groups might partly result from different gene-environmental interactions.
\end{abstract}

\section{Introduction}

Cardiovascular diseases (CVD) are the leading causes of death in global populations, and the burden of CVD in terms of life-years lost, diminished quality of life, and direct and indirect medical costs is enormous [1]. It is well known that dyslipidemia is a major risk factor for CVD [2-5] and that it is the target for therapeutic intervention [6]. Epidemiological studies have consistently shown that dyslipidemia is a complex trait resulted from the joint effects of multiple genetic and environmental causes [7-9]. The heritability estimates of the interindividual variations in serum lipid levels from both twin and family studies are in the range of $40-70 \%$, suggesting a considerable genetic contribution [10, 11]. Therefore, the understanding of the correlation of genetic variants and serum lipid levels may be a promising avenue for exploring prevention and treatment of CVD.
Genome-wide association studies are rapidly unraveling the role of genetic factors in the pathogeneses of dyslipidemia. It was reported that common variants at loci together can explain about $10 \%$ of the variations in each lipid trait $[7$, 12]. Rare variants with large individual effects may also contribute to the heritability of lipid traits [12]. Findings from large-scale studies suggested a strong linkage between the genetic variants at the Krüppel-like factor 14 (KLF14) locus and serum high-density lipoprotein cholesterol (HDLC) concentrations and type 2 diabetes [13-18]. KLF14, which exhibits imprinted expression from the maternal allele in embryonic and extraembryonic tissues, is an intronless member of the KLF family located at chromosome 7q32 [19]. The KLF family of transcription factors is characterized by three highly conserved $\mathrm{Cys}_{2} / \mathrm{His}_{2}$-type zinc fingers which bind to the regulatory regions of genes to mediate activation and/or repression of transcription. Thus far, 17 members 
of the KLF family have been identified and characterized across mammalian systems. These KLF proteins regulate diverse biological processes that include proliferation, differentiation, growth, development, survival, and responses to external stress [20-22]. Recently, increasing evidence suggested that no fewer than eight members of the KLF family have been identified to be key players in the transcription network controlling preadipocyte formation, adipogenesis, lipogenesis, and obesity [23, 24]. The abnormalities caused by excess adipogenesis can result in pathological conditions which are linked to several interrelated diseases such as dyslipidemia and CVD. In an exciting new discovery, the KLF14 is shown to act as a master role in regulating the expression of adipose genes that are associated with key metabolic traits [25]. The single nucleotide polymorphism (SNP) of rs4731702 14kb upstream of KLF14 has implicated a high correlation with HDL-C and CVD [13-15, 26]. However, little is known about the exact impact of this SNP on lipid metabolism.

China is a multiethnic country containing a majority of Han Chinese and 55 ethnic minorities. Many ethnic minorities dwell in Guangxi Zhuang Autonomous Region and they account for more than one third of local total population. Mulao nationality is one of these minorities with population of 207,352 according to the fifth national census statistics of China in 2000. Their principal place of residence is the Luocheng Mulao Autonomous County, Guangxi Zhuang Autonomous Region, People's Republic of China. The history of this minority can be traced back to the Jin Dynasty (AD265-420). A previous study has shown that the genetic relationship between Mulao nationality and other minorities in Guangxi was much closer than that between Mulao and Han or Uyghur nationality [27]. We believed that the Mulao nationality has become a useful subgroup for population genetic studies. However, there were no studies to examine the association of the rs4731702 SNP and serum lipid levels in this population. Thus, the present study was to detect the distribution of rs4731702 SNP and evaluate the association of the SNP and serum lipid levels in the Guangxi Mulao and Han populations.

\section{Materials and Methods}

2.1. Study Population. This study included 727 subjects of Mulao and 740 subjects of Han Chinese who were randomly selected from our previous stratified randomized samples $[28,29]$. All subjects were rural agricultural workers residing in Luocheng Mulao Autonomous County, Guangxi Zhuang Autonomous Region, People's Republic of China. The subjects of Mulao consisted of 323 (44.43\%) males and 404 (55.57\%) females, aged from 16 to 86 years, with a mean age of $52.32 \pm 14.94$ years. The subjects of Han consisted of 318 $(42.97 \%)$ males and $422(57.03 \%)$ females, aged from 16 to 86 years, with a mean age of $52.08 \pm 15.22$ years. Subjects with diseases related to atherosclerosis, CVD, and diabetes or those who were using lipid-lowering medication were excluded from the study. The present study was conducted in accordance with the guidelines set by the Ethics Committee of the First Affiliated Hospital, Guangxi Medical University. Informed consents were obtained from all the subjects prior to their inclusion into the study.

2.2. Epidemiological Survey. Epidemiological survey was carried out using internationally standardized methods [30]. A standard questionnaire collecting the information on demographics, socioeconomic status, and lifestyle factors was obtained from all the subjects. The alcohol information included questions about the number of liangs (about $50 \mathrm{~g}$ ) of rice wine, corn wine, rum, beer, or liquor consumed during the preceding 12 months. Alcohol consumption was classified as groups of grams of alcohol per day: $\leq 25$ and $>25$. Smoking status was categorized into groups of cigarettes per day: $\leq 20$ and $>20$. Anthropometric measurements were obtained by trained personnel of health care centers including height, weight, and waist circumference. Blood pressure of the subjects in a sitting position was measured taking the mean of 3 separated intervals after the subjects had a 5-minute rest using a mercury sphygmomanometer. Body mass index (BMI) was calculated as weight $/$ height $^{2}\left(\mathrm{~kg} / \mathrm{m}^{2}\right)$.

2.3. Biochemical Parameters. Blood samples were obtained in the fasting state. Biochemical parameters including total cholesterol (TC), triglyceride (TG), HDL-C, and low-density lipoprotein cholesterol (LDL-C) were measured by enzymatic methods with commercially available kits. Serum apolipoprotein (Apo) AI and ApoB concentrations were quantified by the immunoturbidimetric immunoassay using a commercial kit [31]. Fasting blood glucose was determined by glucose meter.

2.4. DNA Amplification and Genotyping. The genomic DNA was obtained from peripheral lymphocytes using the phenolchloroform method [32]. Genotyping was carried out by polymerase chain reaction (PCR) amplification followed by restriction enzyme for restriction fragment length polymorphism (RFLP). For the KLF14 rs4731702 SNP analysis, DNA was amplified using the forward primer, $5^{\prime}$ AATCCCAAGGCATCTATC- $3^{\prime}$, and the reverse primer, $5^{\prime}$ CTTGGATTTTGATTACGG-3' (Sangon, Shanghai, People's Republic of China). Each $25 \mu \mathrm{L}$ PCR reaction mixture consisted of $2 \mu \mathrm{L}$ of genomic DNA, $1 \mu \mathrm{L}$ of each primer (10 pmol/L), $12.5 \mu \mathrm{L}$ of $2 \times$ Taq PCR Master Mix (constituent: $20 \mathrm{mM}$ Tris- $\mathrm{HCl}, \mathrm{pH} 8.3,100 \mathrm{mM} \mathrm{KCl}, 3 \mathrm{mM} \mathrm{MgCl}, 0.1 \mathrm{U}$ Taq Polymerase/ $\mu \mathrm{L}, 500 \mu \mathrm{M}$ dNTP each; Sangon, Shanghai, People's Republic of China), and $8.5 \mu \mathrm{L}$ of $\mathrm{ddH}_{2} \mathrm{O}$ (DNase/RNase-free). The cycle parameters were as follows: 1 cycle at $94^{\circ} \mathrm{C}$ for 5 minutes for an initial denaturation followed by 35 cycles of denaturation for 45 seconds at $94^{\circ} \mathrm{C}$, primer annealing for 45 seconds at $53^{\circ} \mathrm{C}$, primer extension for 45 seconds at $72^{\circ} \mathrm{C}$ and a final extension for 7 minutes at $72^{\circ} \mathrm{C}$. For the restriction digestion, $5 \mu \mathrm{L}$ of amplification products and $5 \mathrm{U}$ of BselI restriction enzyme (Fermentas Co. Canada) were added to each reaction mix, and samples were digested at $65^{\circ} \mathrm{C}$ overnight. Then, the digested fragments were separated by electrophoresis on $2 \%$ agarose gels stained with ethidium bromide and photographed in ultraviolet light. 
TABLE 1: Comparison of demographics, lifestyle, and serum lipid levels between the Mulao and Han populations.

\begin{tabular}{|c|c|c|c|c|}
\hline Parameter & Mulao & Han & $t\left(\chi^{2}\right)$ & $P$ \\
\hline Number & 727 & 740 & - & - \\
\hline Male/female & $323 / 404$ & $318 / 422$ & 0.316 & 0.574 \\
\hline Age (years) & $52.32 \pm 14.94$ & $52.08 \pm 15.22$ & 0.307 & 0.759 \\
\hline Height (cm) & $155.42 \pm 7.97$ & $154.31 \pm 8.00$ & 2.67 & 0.008 \\
\hline Weight (kg) & $52.78 \pm 9.25$ & $53.57 \pm 9.06$ & -1.666 & 0.096 \\
\hline Body mass index $\left(\mathrm{kg} / \mathrm{m}^{2}\right)$ & $21.79 \pm 3.08$ & $22.48 \pm 3.41$ & -4.034 & $<0.001$ \\
\hline Waist circumference & $75.13 \pm 8.95$ & $75.41 \pm 7.90$ & -0.647 & 0.518 \\
\hline \multicolumn{5}{|l|}{ Cigarette smoking $[n(\%)]$} \\
\hline Nonsmoker & $545(75.0)$ & $531(71.8)$ & & \\
\hline$\leq 20$ cigarettes/day & $155(21.3)$ & $183(24.7)$ & 2.406 & 0.300 \\
\hline$>20$ cigarettes/day & $27(3.7)$ & $26(3.5)$ & & \\
\hline \multicolumn{5}{|l|}{ Alcohol consumption $[n(\%)]$} \\
\hline Nondrinker & $553(76.1)$ & $576(77.8)$ & & \\
\hline$\leq 25 \mathrm{~g} /$ day & $59(8.1)$ & $76(10.3)$ & 6.086 & 0.048 \\
\hline$>25$ g/day & $115(15.8)$ & 88 (11.9) & & \\
\hline Systolic blood pressure (mmHg) & $129.56 \pm 22.01$ & $129.74 \pm 19.17$ & -0.168 & 0.867 \\
\hline Diastolic blood pressure ( $\mathrm{mmHg}$ ) & $81.01 \pm 11.54$ & $82.22 \pm 11.18$ & -2.045 & 0.041 \\
\hline Pulse pressure $(\mathrm{mmHg})$ & $48.55 \pm 16.59$ & $47.52 \pm 14.48$ & 1.271 & 0.204 \\
\hline Blood glucose (mmol/L) & $6.02 \pm 1.64$ & $6.06 \pm 1.77$ & -0.441 & 0.659 \\
\hline Total cholesterol (mmol/L) & $5.07 \pm 1.34$ & $5.00 \pm 1.10$ & 1.071 & 0.284 \\
\hline Triglyceride (mmol/L) & $1.07(0.78)$ & $1.08(0.88)$ & -0.996 & 0.319 \\
\hline HDL-C (mmol/L) & $1.75 \pm 0.46$ & $1.72 \pm 0.53$ & 1.345 & 0.179 \\
\hline LDL-C (mmol/L) & $2.95 \pm 0.90$ & $2.86 \pm 0.85$ & 2.048 & 0.041 \\
\hline Apolipoprotein (Apo) AI (g/L) & $1.32 \pm 0.40$ & $1.33 \pm 0.27$ & -0.497 & 0.619 \\
\hline $\operatorname{ApoB}(g / L)$ & $0.98 \pm 0.55$ & $0.86 \pm 0.21$ & 5.828 & $<0.001$ \\
\hline ApoAI/ApoB & $1.57 \pm 0.75$ & $1.64 \pm 0.51$ & -2.016 & 0.044 \\
\hline
\end{tabular}

HDL-C: high-density lipoprotein cholesterol; LDL-C: low-density lipoprotein cholesterol; ApoAI: apolipoprotein AI; ApoB: apolipoprotein B; ApoAI/ApoB: the ratio of apolipoprotein AI to apolipoprotein $B$. The value of triglyceride was presented as median (interquartile range), and the difference between the two ethnic groups was determined by the Wilcoxon-Mann-Whitney test.

TABLE 2: Comparison of the genotypic and allelic frequencies of KLF14 rs4731702 SNP between the Mulao and Han populations ( $n$ (\%)).

\begin{tabular}{|c|c|c|c|c|c|c|}
\hline \multirow{2}{*}{ Group } & \multirow{2}{*}{$n$} & \multicolumn{3}{|c|}{ Genotype } & \multicolumn{2}{|c|}{ Allele } \\
\hline & & $\mathrm{CC}$ & CT & TT & $\mathrm{C}$ & $\mathrm{T}$ \\
\hline Mulao & 727 & $319(44.5)$ & $297(40.0)$ & 111 (15.5) & $935(64.3)$ & $519(35.7)$ \\
\hline Han & 740 & $348(47.0)$ & $290(39.2)$ & $102(13.8)$ & $986(66.6)$ & $494(33.4)$ \\
\hline$\chi^{2}$ & - & & 1.294 & & & \\
\hline$P$ & - & & 0.524 & & & \\
\hline \multicolumn{7}{|l|}{ Mulao } \\
\hline Male & 323 & $131(40.6)$ & $140(43.3)$ & $52(16.1)$ & $402(62.2)$ & $244(37.8)$ \\
\hline Female & 404 & $188(46.5)$ & $157(38.9)$ & 59 (14.6) & $533(66.0)$ & $275(34.0)$ \\
\hline$\chi^{2}$ & - & & 2.607 & & & \\
\hline$P$ & - & & 0.272 & & & \\
\hline \multicolumn{7}{|l|}{ Han } \\
\hline Male & 318 & $156(49.1)$ & $132(41.5)$ & $30(9.4)$ & $444(69.8)$ & $192(30.2)$ \\
\hline Female & 422 & $192(45.5)$ & $158(37.4)$ & $72(17.1)$ & $542(64.2)$ & $302(35.8)$ \\
\hline$\chi^{2}$ & - & & 8.909 & & & \\
\hline$P$ & - & & 0.012 & & & \\
\hline
\end{tabular}


TABLE 3: The KLF14 rs4731702 genotypes and serum lipid levels between the Mulao and Han populations.

\begin{tabular}{|c|c|c|c|c|c|c|c|c|}
\hline Genotype & $n$ & $\mathrm{TC}(\mathrm{mmol} / \mathrm{L})$ & $\mathrm{TG}(\mathrm{mmol} / \mathrm{L})$ & HDL-C (mmol/L) & LDL-C (mmol/L) & ApoAI (g/L) & ApoB (g/L) & ApoAI/ApoB \\
\hline \multicolumn{9}{|l|}{ Mulao } \\
\hline CC & 319 & $5.00 \pm 1.30$ & $1.03(0.82)$ & $1.71 \pm 0.42$ & $2.86 \pm 0.88$ & $1.28 \pm 0.41$ & $0.96 \pm 0.54$ & $1.52 \pm 0.63$ \\
\hline $\mathrm{CT}$ & 297 & $5.16 \pm 1.44$ & $1.09(0.75)$ & $1.79 \pm 0.48$ & $3.00 \pm 0.94$ & $1.36 \pm 0.40$ & $1.01 \pm 0.56$ & $1.59 \pm 0.83$ \\
\hline $\mathrm{TT}$ & 111 & $5.07 \pm 1.15$ & $1.15(0.75)$ & $1.77 \pm 0.46$ & $3.03 \pm 0.83$ & $1.37 \pm 0.40$ & $0.96 \pm 0.53$ & $1.67 \pm 0.85$ \\
\hline$F$ & - & 1.084 & 0.637 & 2.392 & 3.077 & 4.145 & 0.817 & 1.833 \\
\hline$P$ & - & 0.339 & 0.727 & 0.092 & 0.047 & 0.016 & 0.442 & 0.161 \\
\hline \multicolumn{9}{|c|}{ Mulao/male } \\
\hline $\mathrm{CC}$ & 131 & $5.06 \pm 1.58$ & $1.21(1.00)$ & $1.66 \pm 0.40$ & $2.74 \pm 0.77$ & $1.24 \pm 0.44$ & $1.01 \pm 0.59$ & $1.41 \pm 0.64$ \\
\hline $\mathrm{CT}$ & 140 & $5.37 \pm 1.55$ & $1.17(0.96)$ & $1.81 \pm 0.56$ & $3.01 \pm 0.73$ & $1.38 \pm 0.42$ & $1.08 \pm 0.63$ & $1.51 \pm 0.67$ \\
\hline $\mathrm{TT}$ & 52 & $5.12 \pm 0.93$ & $1.07(1.17)$ & $1.78 \pm 0.51$ & $2.99 \pm 0.12$ & $1.44 \pm 0.39$ & $0.96 \pm 0.48$ & $1.66 \pm 0.65$ \\
\hline$F$ & - & 1.526 & 0.598 & 3.16 & 4.583 & 6.023 & 0.947 & 2.603 \\
\hline$P$ & - & 0.219 & 0.742 & 0.044 & 0.011 & 0.003 & 0.389 & 0.076 \\
\hline \multicolumn{9}{|c|}{ Mulao/female } \\
\hline CC & 188 & $4.95 \pm 1.05$ & $0.99(0.68)$ & $1.75 \pm 0.44$ & $2.91 \pm 0.85$ & $1.30 \pm 0.38$ & $0.93 \pm 0.49$ & $1.59 \pm 0.61$ \\
\hline $\mathrm{CT}$ & 157 & $4.97 \pm 1.32$ & $0.99(0.63)$ & $1.78 \pm 0.41$ & $3.03 \pm 0.99$ & $1.34 \pm 0.37$ & $0.95 \pm 0.49$ & $1.67 \pm 0.95$ \\
\hline $\mathrm{TT}$ & 59 & $5.02 \pm 1.32$ & $1.18(0.66)$ & $1.76 \pm 0.42$ & $3.04 \pm 0.96$ & $1.31 \pm 0.40$ & $0.96 \pm 0.58$ & $1.69 \pm 1.00$ \\
\hline$F$ & - & 0.073 & 3.986 & 0.205 & 0.849 & 0.349 & 0.177 & 0.468 \\
\hline$P$ & - & 0.929 & 0.136 & 0.814 & 0.429 & 0.705 & 0.838 & 0.626 \\
\hline \multicolumn{9}{|l|}{ Han } \\
\hline CC & 348 & $4.99 \pm 0.91$ & $1.10(0.90)$ & $1.77 \pm 0.64$ & $2.81 \pm 0.76$ & $1.35 \pm 0.27$ & $0.84 \pm 0.19$ & $1.69 \pm 0.53$ \\
\hline $\mathrm{CT}$ & 290 & $4.96 \pm 1.33$ & $1.06(0.88)$ & $1.65 \pm 0.42$ & $2.84 \pm 0.91$ & $1.30 \pm 0.26$ & $0.86 \pm 0.24$ & $1.59 \pm 0.48$ \\
\hline $\mathrm{TT}$ & 102 & $5.19 \pm 0.92$ & $1.07(1.12)$ & $1.74 \pm 0.40$ & $3.04 \pm 0.92$ & $1.36 \pm 0.25$ & $0.88 \pm 0.17$ & $1.60 \pm 0.45$ \\
\hline$F$ & - & 1.638 & 1.071 & 4.283 & 2.961 & 2.942 & 1.605 & 3.727 \\
\hline$P$ & - & 0.195 & 0.585 & 0.014 & 0.052 & 0.056 & 0.201 & 0.025 \\
\hline \multicolumn{9}{|l|}{ Han/male } \\
\hline $\mathrm{CC}$ & 156 & $5.23 \pm 0.77$ & $1.34(0.89)$ & $1.72 \pm 0.45$ & $2.94 \pm 0.75$ & $1.40 \pm 0.32$ & $0.89 \pm 0.16$ & $1.64 \pm 0.54$ \\
\hline $\mathrm{CT}$ & 132 & $5.22 \pm 1.54$ & 1.09 (1.06) & $1.64 \pm 0.41$ & $2.92 \pm 0.94$ & $1.33 \pm 0.26$ & $0.95 \pm 0.25$ & $1.47 \pm 0.38$ \\
\hline $\mathrm{TT}$ & 30 & $5.25 \pm 0.72$ & $1.33(1.52)$ & $1.62 \pm 0.28$ & $2.97 \pm 0.87$ & $1.32 \pm 0.18$ & $0.92 \pm 0.14$ & $1.47 \pm 0.28$ \\
\hline$F$ & - & 0.014 & 5.803 & 1.749 & 0.042 & 3.147 & 2.478 & 5.353 \\
\hline$P$ & - & 0.986 & 0.055 & 0.176 & 0.959 & 0.044 & 0.086 & 0.005 \\
\hline \multicolumn{9}{|c|}{ Han/female } \\
\hline CC & 192 & $4.79 \pm 0.98$ & $0.97(0.84)$ & $1.81 \pm 0.75$ & $2.75 \pm 0.81$ & $1.30 \pm 0.23$ & $0.80 \pm 0.20$ & $1.74 \pm 0.52$ \\
\hline $\mathrm{CT}$ & 158 & $4.75 \pm 1.10$ & $1.05(0.74)$ & $1.66 \pm 0.44$ & $2.77 \pm 0.89$ & $1.28 \pm 0.27$ & $0.79 \pm 0.20$ & $1.69 \pm 0.54$ \\
\hline $\mathrm{TT}$ & 72 & $5.16 \pm 1.00$ & $1.05(0.75)$ & $1.78 \pm 0.44$ & $3.08 \pm 0.95$ & $1.37 \pm 0.27$ & $0.87 \pm 0.19$ & $1.66 \pm 0.49$ \\
\hline$F$ & - & 4.317 & 2.708 & 2.965 & 4.037 & 3.511 & 3.947 & 0.707 \\
\hline$P$ & - & 0.014 & 0.258 & 0.052 & 0.018 & 0.031 & 0.020 & 0.494 \\
\hline
\end{tabular}

TC: total cholesterol; TG: triglyceride; HDL-C: high-density lipoprotein cholesterol; LDL-C: low-density lipoprotein cholesterol; ApoAI: apolipoprotein AI; ApoB: apolipoprotein B; ApoAI/ApoB: the ratio of apolipoprotein AI to apolipoprotein B. The values of TG were presented as median (interquartile range). The difference among the genotypes was determined by the Kruskal-Wallis test.

Genotypes were scored by an experienced reader blinded to the epidemiological data and serum lipid levels.

2.5. DNA Sequencing. Three samples detected by the PCRRFLP were also confirmed by direct sequencing with an ABI Prism 3100 (Applied Biosystems) in Shanghai Sangon Biological Engineering Technology \& Services Co., Ltd., People's Republic of China.

2.6. Diagnostic Criteria. The normal values of serum TC, TG, HDL-C, LDL-C, ApoAI, ApoB levels, and the ratio of
ApoAI to ApoB in our Clinical Science Experiment Center were $3.10-5.17,0.56-1.70,0.91-1.81,2.70-3.20 \mathrm{mmol} / \mathrm{L}, 1.00-$ $1.78,0.63-1.14 \mathrm{~g} / \mathrm{L}$, and 1.00-2.50, respectively [32]. Hypertension was assessed according to the criteria of 1999 World Health Organization-International Society of Hypertension Guidelines for the management of hypertension [33]. Normal weight, overweight, and obesity were defined as a BMI $<24$, $24-28$, and $>28 \mathrm{~kg} / \mathrm{m}^{2}$, respectively [34].

2.7. Statistical Analyses. Statistical analyses were performed by the statistical software package SPSS 16.0 (SPSS Inc., Chicago, Illinois). Qualitative variables were expressed as 
TABLE 4: Relationship between serum lipid parameters and relative factors in the Mulao and Han populations.

\begin{tabular}{|c|c|c|c|c|c|c|}
\hline Lipid parameter & Risk factor & $\begin{array}{c}\text { Unstandardized } \\
\text { coefficient }\end{array}$ & Std. error & $\begin{array}{c}\text { Standardized } \\
\text { coefficient }\end{array}$ & $t$ & $P$ \\
\hline \multicolumn{7}{|l|}{ Mulao and Han } \\
\hline \multirow{4}{*}{$\mathrm{TC}$} & Waist circumference & 0.020 & 0.004 & 0.135 & 5.142 & 0.000 \\
\hline & Alcohol consumption & 0.011 & 0.002 & 0.139 & 5.339 & 0.000 \\
\hline & Age & 0.236 & 0.044 & 0.138 & 5.363 & 0.000 \\
\hline & Diastolic blood pressure & 0.008 & 0.003 & 0.071 & 2.643 & 0.008 \\
\hline \multirow{4}{*}{ TG } & Waist circumference & 0.063 & 0.007 & 0.220 & 8.631 & 0.000 \\
\hline & Alcohol consumption & 0.331 & 0.093 & 0.100 & 3.568 & 0.000 \\
\hline & Cigarette smoking & 0.113 & 0.035 & 0.082 & 3.270 & 0.001 \\
\hline & Blood glucose & 0.396 & 0.123 & 0.090 & 3.227 & 0.001 \\
\hline \multirow{4}{*}{ HDL-C } & Waist circumference & -0.009 & 0.002 & -0.145 & -4.028 & 0.000 \\
\hline & Alcohol consumption & 0.117 & 0.021 & 0.168 & 5.514 & 0.000 \\
\hline & Gender & 0.090 & 0.031 & 0.090 & 2.889 & 0.004 \\
\hline & Body mass index & -0.014 & 0.005 & -0.091 & -2.607 & 0.009 \\
\hline \multirow{4}{*}{ LDL-C } & Body mass index & 0.052 & 0.007 & 0.193 & 7.642 & 0.000 \\
\hline & Age & 0.010 & 0.001 & 0.173 & 6.872 & 0.000 \\
\hline & Genotype & 0.089 & 0.031 & 0.072 & 2.867 & 0.004 \\
\hline & Ethnic group & -0.123 & 0.044 & -0.070 & -2.765 & 0.006 \\
\hline \multirow{3}{*}{ ApoAI } & Alcohol consumption & 0.127 & 0.015 & 0.266 & 8.722 & 0.000 \\
\hline & Waist circumference & -0.004 & 0.001 & -0.086 & -3.286 & 0.001 \\
\hline & Gender & 0.051 & 0.021 & 0.074 & 2.385 & 0.017 \\
\hline \multirow{6}{*}{ ApoB } & Waist circumference & 0.007 & 0.002 & 0.133 & 3.724 & 0.000 \\
\hline & Ethnic group & -0.135 & 0.021 & -0.161 & -6.410 & 0.000 \\
\hline & Blood glucose & 0.023 & 0.006 & 0.092 & 3.624 & 0.000 \\
\hline & Gender & -0.058 & 0.022 & -0.069 & -2.648 & 0.008 \\
\hline & Systolic blood pressure & 0.001 & 0.001 & 0.058 & 2.247 & 0.025 \\
\hline & Body mass index & 0.009 & 0.004 & 0.069 & 1.973 & 0.049 \\
\hline \multirow{7}{*}{ ApoAI/ApoB } & Waist circumference & -0.011 & 0.003 & -0.147 & -4.130 & 0.000 \\
\hline & Age & -0.003 & 0.001 & -0.071 & -2.741 & 0.006 \\
\hline & Body mass index & -0.024 & 0.007 & -0.122 & -3.511 & 0.000 \\
\hline & Ethnic group & 0.092 & 0.032 & 0.072 & 2.856 & 0.004 \\
\hline & Blood glucose & -0.025 & 0.010 & -0.067 & -2.577 & 0.010 \\
\hline & Alcohol consumption & 0.121 & 0.027 & 0.135 & 4.505 & 0.000 \\
\hline & Gender & 0.175 & 0.039 & 0.135 & 4.445 & 0.000 \\
\hline \multicolumn{7}{|l|}{ Mulao } \\
\hline \multirow{3}{*}{$\mathrm{TC}$} & Body mass index & 0.067 & 0.016 & 0.155 & 4.298 & 0.000 \\
\hline & Alcohol consumption & 0.251 & 0.065 & 0.140 & 3.871 & 0.000 \\
\hline & Age & 0.011 & 0.003 & 0.119 & 3.289 & 0.001 \\
\hline \multirow{2}{*}{ TG } & Waist circumference & 0.054 & 0.010 & 0.202 & 5.575 & 0.000 \\
\hline & Alcohol consumption & 0.497 & 0.113 & 0.160 & 4.418 & 0.000 \\
\hline \multirow{3}{*}{ HDL-C } & Body mass index & -0.037 & 0.005 & -0.248 & -6.947 & 0.000 \\
\hline & Alcohol consumption & 0.118 & 0.027 & 0.194 & 4.443 & 0.000 \\
\hline & Gender & 0.105 & 0.040 & 0.115 & 2.631 & 0.009 \\
\hline \multirow{3}{*}{ LDL-C } & Body mass index & 0.054 & 0.011 & 0.185 & 5.121 & 0.000 \\
\hline & Age & 0.008 & 0.002 & 0.125 & 3.449 & 0.001 \\
\hline & Genotype & 0.101 & 0.046 & 0.080 & 2.203 & 0.028 \\
\hline
\end{tabular}


TABLE 4: Continued.

\begin{tabular}{|c|c|c|c|c|c|c|}
\hline Lipid parameter & Risk factor & $\begin{array}{c}\text { Unstandardized } \\
\text { coefficient }\end{array}$ & Std. error & $\begin{array}{c}\text { Standardized } \\
\text { coefficient }\end{array}$ & $t$ & $P$ \\
\hline \multirow{3}{*}{ ApoAI } & Alcohol consumption & 0.128 & 0.024 & 0.237 & 5.330 & 0.000 \\
\hline & Gender & 0.096 & 0.036 & 0.119 & 2.677 & 0.008 \\
\hline & Genotype & 0.049 & 0.020 & 0.088 & 2.404 & 0.016 \\
\hline \multirow{2}{*}{ ApoB } & Waist circumference & 0.011 & 0.002 & 0.177 & 4.801 & 0.000 \\
\hline & Blood glucose & 0.027 & 0.012 & 0.081 & 2.187 & 0.029 \\
\hline ApoAI/ApoB & Waist circumference & -0.018 & 0.003 & -0.206 & -5.680 & 0.000 \\
\hline \multicolumn{7}{|l|}{ Han } \\
\hline \multirow{5}{*}{ TC } & Diastolic blood pressure & 0.019 & 0.004 & 0.192 & 5.170 & 0.000 \\
\hline & Alcohol consumption & 0.249 & 0.057 & 0.154 & 4.407 & 0.000 \\
\hline & Age & 0.009 & 0.003 & 0.124 & 3.334 & 0.001 \\
\hline & Waist circumference & 0.017 & 0.005 & 0.121 & 3.338 & 0.001 \\
\hline & Blood glucose & 0.053 & 0.022 & 0.085 & 2.374 & 0.018 \\
\hline \multirow{6}{*}{ TG } & Waist circumference & 0.081 & 0.014 & 0.268 & 5.796 & 0.000 \\
\hline & Cigarette smoking & 0.903 & 0.151 & 0.203 & 5.964 & 0.000 \\
\hline & Blood glucose & 0.235 & 0.048 & 0.174 & 4.918 & 0.000 \\
\hline & Diastolic blood pressure & 0.035 & 0.008 & 0.164 & 4.461 & 0.000 \\
\hline & Age & -0.019 & 0.006 & -0.118 & -3.209 & 0.001 \\
\hline & Body mass index & -0.067 & 0.032 & -0.095 & -2.078 & 0.038 \\
\hline \multirow{2}{*}{ HDL-C } & Waist circumference & -0.014 & 0.002 & -0.206 & -5.615 & 0.000 \\
\hline & Alcohol consumption & 0.075 & 0.029 & 0.095 & 2.597 & 0.010 \\
\hline \multirow{4}{*}{ LDL-C } & Age & 0.012 & 0.002 & 0.221 & 6.364 & 0.000 \\
\hline & Body mass index & 0.045 & 0.009 & 0.182 & 5.141 & 0.000 \\
\hline & Cigarette smoking & -0.310 & 0.069 & -0.196 & -4.480 & 0.000 \\
\hline & Gender & -0.292 & 0.076 & -0.170 & -3.840 & 0.000 \\
\hline \multirow{6}{*}{ ApoAI } & Genotype & 0.087 & 0.042 & 0.072 & 2.073 & 0.039 \\
\hline & Alcohol consumption & 0.116 & 0.017 & 0.295 & 7.001 & 0.000 \\
\hline & Body mass index & -0.012 & 0.003 & -0.152 & -4.304 & 0.000 \\
\hline & Cigarette smoking & 0.075 & 0.022 & 0.151 & 3.350 & 0.001 \\
\hline & Gender & 0.067 & 0.025 & 0.124 & 2.651 & 0.008 \\
\hline & Waist circumference & 0.005 & 0.001 & 0.193 & 4.302 & 0.000 \\
\hline \multirow{5}{*}{ ApoB } & Blood glucose & 0.019 & 0.004 & 0.166 & 4.946 & 0.000 \\
\hline & Alcohol consumption & 0.030 & 0.012 & 0.097 & 2.548 & 0.011 \\
\hline & Body mass index & 0.009 & 0.003 & 0.151 & 3.471 & 0.001 \\
\hline & Gender & -0.046 & 0.016 & -0.111 & -2.849 & 0.005 \\
\hline & Diastolic blood pressure & 0.002 & 0.001 & 0.110 & 3.137 & 0.002 \\
\hline \multirow{9}{*}{ ApoAI/ApoB } & Age & 0.001 & 0.000 & 0.093 & 2.656 & 0.008 \\
\hline & Waist circumference & -0.009 & 0.003 & -0.148 & -3.190 & 0.001 \\
\hline & Body mass index & -0.031 & 0.007 & -0.211 & -4.675 & 0.000 \\
\hline & Age & -0.004 & 0.001 & -0.108 & -3.103 & 0.002 \\
\hline & Blood glucose & -0.022 & 0.010 & -0.078 & -2.228 & 0.026 \\
\hline & Alcohol consumption & 0.091 & 0.030 & 0.122 & 3.009 & 0.003 \\
\hline & Gender & 0.230 & 0.047 & 0.225 & 4.868 & 0.000 \\
\hline & Cigarette smoking & 0.127 & 0.041 & 0.134 & 3.084 & 0.002 \\
\hline & Genotype & -0.052 & 0.024 & -0.072 & -2.144 & 0.032 \\
\hline
\end{tabular}

TC: total cholesterol; TG: triglyceride; HDL-C: high-density lipoprotein cholesterol; LDL-C: low-density lipoprotein cholesterol; ApoAI: apolipoprotein AI; ApoB: apolipoprotein B; ApoAI/ApoB: the ratio of apolipoprotein AI to apolipoprotein B. 
TABle 5: Relationship between serum lipid parameters and relative factors in males and females of the Mulao and Han populations.

\begin{tabular}{|c|c|c|c|c|c|c|}
\hline Lipid parameter & Risk factor & $\begin{array}{c}\text { Unstandardized } \\
\text { coefficient }\end{array}$ & Std. error & $\begin{array}{c}\text { Standardized } \\
\text { coefficient }\end{array}$ & $t$ & $P$ \\
\hline \multicolumn{7}{|l|}{ Mulao/male } \\
\hline \multirow{2}{*}{ TC } & Body mass index & 0.084 & 0.026 & 0.174 & 3.183 & 0.002 \\
\hline & Alcohol consumption & 0.251 & 0.090 & 0.152 & 2.790 & 0.006 \\
\hline \multirow{2}{*}{ TG } & Waist circumference & 0.082 & 0.020 & 0.227 & 4.181 & 0.000 \\
\hline & Alcohol consumption & 0.412 & 0.194 & 0.115 & 2.119 & 0.035 \\
\hline \multirow{2}{*}{ HDL-C } & Alcohol consumption & 0.124 & 0.029 & 0.227 & 4.231 & 0.000 \\
\hline & Body mass index & -0.036 & 0.009 & -0.226 & -4.218 & 0.000 \\
\hline \multirow{2}{*}{ LDL-C } & Body mass index & 0.045 & 0.016 & 0.157 & 2.828 & 0.005 \\
\hline & Genotype & 0.154 & 0.068 & 0.126 & 2.257 & 0.025 \\
\hline \multirow{2}{*}{ ApoAI } & Alcohol consumption & 0.127 & 0.025 & 0.264 & 4.978 & 0.000 \\
\hline & Genotype & 0.102 & 0.032 & 0.168 & 3.167 & 0.002 \\
\hline ApoB & Waist circumference & 0.008 & 0.004 & 0.127 & 2.301 & 0.022 \\
\hline \multirow{2}{*}{ ApoAI/ApoB } & Waist circumference & -0.016 & 0.004 & -0.217 & -4.003 & 0.000 \\
\hline & Alcohol consumption & 0.136 & 0.040 & 0.185 & 3.418 & 0.001 \\
\hline \multicolumn{7}{|l|}{ Mulao/female } \\
\hline \multirow{2}{*}{$\mathrm{TC}$} & Age & 0.015 & 0.004 & 0.181 & 3.714 & 0.000 \\
\hline & Body mass index & 0.054 & 0.019 & 0.138 & 2.835 & 0.005 \\
\hline TG & Waist circumference & 0.026 & 0.007 & 0.194 & 3.968 & 0.000 \\
\hline HDL-C & Body mass index & -0.038 & 0.007 & -0.273 & -5.687 & 0.000 \\
\hline \multirow{2}{*}{ LDL-C } & Body mass index & 0.064 & 0.014 & 0.213 & 4.477 & 0.000 \\
\hline & Age & 0.013 & 0.003 & 0.211 & 4.425 & 0.000 \\
\hline \multirow{4}{*}{ ApoB } & Waist circumference & 0.013 & 0.003 & 0.209 & 4.356 & 0.000 \\
\hline & Cigarette smoking & 1.198 & 0.342 & 0.167 & 3.504 & 0.001 \\
\hline & Blood glucose & 0.037 & 0.018 & 0.102 & 2.079 & 0.038 \\
\hline & Age & 0.003 & 0.002 & 0.097 & 1.987 & 0.048 \\
\hline \multirow{2}{*}{ ApoAI/ApoB } & Waist circumference & -0.019 & 0.005 & -0.182 & -3.736 & 0.000 \\
\hline & Age & -0.008 & 0.003 & -0.140 & -2.878 & 0.004 \\
\hline \multicolumn{7}{|l|}{ Han/male } \\
\hline \multirow{2}{*}{$\mathrm{TC}$} & Diastolic blood pressure & 0.032 & 0.005 & 0.312 & 5.908 & 0.000 \\
\hline & Alcohol consumption & 0.213 & 0.071 & 0.158 & 2.988 & 0.003 \\
\hline \multirow{5}{*}{ TG } & Waist circumference & 0.079 & 0.022 & 0.194 & 3.569 & 0.000 \\
\hline & Cigarette smoking & 1.254 & 0.280 & 0.235 & 4.482 & 0.000 \\
\hline & Blood glucose & 0.440 & 0.106 & 0.228 & 4.141 & 0.000 \\
\hline & Diastolic blood pressure & 0.055 & 0.016 & 0.189 & 3.466 & 0.001 \\
\hline & Age & -0.024 & 0.011 & -0.116 & -2.077 & 0.039 \\
\hline \multirow{4}{*}{ HDL-C } & Waist circumference & -0.015 & 0.003 & -0.287 & -5.446 & 0.000 \\
\hline & Alcohol consumption & 0.100 & 0.026 & 0.202 & 3.842 & 0.000 \\
\hline & Genotype & -0.073 & 0.034 & -0.113 & -2.163 & 0.031 \\
\hline & Blood glucose & -0.027 & 0.013 & -0.108 & -2.052 & 0.041 \\
\hline \multirow{2}{*}{ LDL-C } & Cigarette smoking & -0.330 & 0.074 & -0.241 & -4.479 & 0.000 \\
\hline & Body mass index & 0.040 & 0.012 & 0.180 & 3.351 & 0.001 \\
\hline \multirow{4}{*}{ ApoAI } & Alcohol consumption & 0.120 & 0.017 & 0.358 & 6.938 & 0.000 \\
\hline & Body mass index & -0.012 & 0.004 & -0.160 & -3.146 & 0.002 \\
\hline & Cigarette smoking & 0.072 & 0.024 & 0.157 & 3.021 & 0.003 \\
\hline & Genotype & -0.059 & 0.022 & -0.137 & -2.725 & 0.007 \\
\hline
\end{tabular}


TABLE 5: Continued.

\begin{tabular}{|c|c|c|c|c|c|c|}
\hline Lipid parameter & Risk factor & $\begin{array}{c}\text { Unstandardized } \\
\text { coefficient }\end{array}$ & Std. error & $\begin{array}{c}\text { Standardized } \\
\text { coefficient }\end{array}$ & $t$ & $P$ \\
\hline \multirow{5}{*}{ ApoB } & Waist circumference & 0.005 & 0.002 & 0.202 & 3.283 & 0.001 \\
\hline & Diastolic blood pressure & 0.004 & 0.001 & 0.210 & 4.062 & 0.000 \\
\hline & Blood glucose & 0.019 & 0.006 & 0.158 & 3.136 & 0.002 \\
\hline & Alcohol consumption & 0.032 & 0.012 & 0.135 & 2.683 & 0.008 \\
\hline & Body mass index & 0.007 & 0.003 & 0.136 & 2.219 & 0.027 \\
\hline \multirow{5}{*}{ ApoAI/ApoB } & Body mass index & -0.027 & 0.007 & -0.220 & -3.621 & 0.000 \\
\hline & Alcohol consumption & 0.102 & 0.028 & 0.184 & 3.610 & 0.000 \\
\hline & Waist circumference & -0.012 & 0.004 & -0.204 & -3.372 & 0.001 \\
\hline & Genotype & -0.124 & 0.036 & -0.173 & -3.485 & 0.001 \\
\hline & Cigarette smoking & 0.113 & 0.039 & 0.148 & 2.889 & 0.004 \\
\hline \multicolumn{7}{|l|}{ Han/female } \\
\hline \multirow{3}{*}{ TC } & Age & 0.023 & 0.003 & 0.325 & 6.993 & 0.000 \\
\hline & Body mass index & 0.061 & 0.016 & 0.176 & 3.925 & 0.000 \\
\hline & Blood glucose & 0.054 & 0.026 & 0.096 & 2.069 & 0.039 \\
\hline \multirow{3}{*}{ TG } & Waist circumference & 0.046 & 0.008 & 0.265 & 5.645 & 0.000 \\
\hline & Blood glucose & 0.107 & 0.032 & 0.153 & 3.370 & 0.001 \\
\hline & Diastolic blood pressure & 0.016 & 0.005 & 0.138 & 2.942 & 0.003 \\
\hline HDL-C & Waist circumference & -0.011 & 0.004 & -0.129 & -2.669 & 0.008 \\
\hline \multirow{4}{*}{ LDL-C } & Age & 0.020 & 0.003 & 0.342 & 7.574 & 0.000 \\
\hline & Waist circumference & 0.021 & 0.005 & 0.183 & 4.071 & 0.000 \\
\hline & Alcohol consumption & -0.008 & 0.004 & -0.097 & -2.155 & 0.032 \\
\hline & Genotype & 0.109 & 0.052 & 0.095 & 2.120 & 0.035 \\
\hline \multirow{2}{*}{ ApoAI } & Alcohol consumption & -0.012 & 0.004 & -0.138 & -2.852 & 0.005 \\
\hline & Body mass index & 0.003 & 0.001 & 0.114 & 2.353 & 0.019 \\
\hline \multirow{4}{*}{ ApoB } & Waist circumference & 0.005 & 0.002 & 0.187 & 2.647 & 0.008 \\
\hline & Blood glucose & 0.021 & 0.005 & 0.194 & 4.254 & 0.000 \\
\hline & Age & 0.002 & 0.001 & 0.146 & 2.969 & 0.003 \\
\hline & Body mass index & 0.010 & 0.005 & 0.155 & 2.202 & 0.028 \\
\hline \multirow{4}{*}{ ApoAI/ApoB } & Age & -0.007 & 0.002 & -0.207 & -4.291 & 0.000 \\
\hline & Body mass index & -0.050 & 0.008 & -0.285 & -6.316 & 0.000 \\
\hline & Cigarette smoking & 0.043 & 0.012 & 0.165 & 3.531 & 0.000 \\
\hline & Blood glucose & -0.031 & 0.013 & -0.110 & -2.343 & 0.020 \\
\hline
\end{tabular}

TC: total cholesterol; TG: triglyceride; HDL-C: high-density lipoprotein cholesterol; LDL-C: low-density lipoprotein cholesterol; ApoAI: Apolipoprotein AI; ApoB: Apolipoprotein B; ApoAI/ApoB: the ratio of Apolipoprotein AI to Apolipoprotein B.

raw count and percentage. The quantitative variables were presented as mean \pm standard deviation (serum TG levels were presented as medians and interquartile ranges). General characteristics between Mulao and Han were compared by Student's unpaired $t$-test. Genotypic and allelic frequencies were calculated by direct counting, and the standard goodness-of-fit test was used to investigate departures from Hardy-Weinberg equilibrium. The difference in genotype distribution and sex ratio between the populations was tested by chi-square analysis. The analysis of covariance (ANCOVA) was performed to estimate the association of genotypes and serum lipid parameters. Factors that may influence serum lipid concentrations such as sex, age, BMI, blood pressure, alcohol consumption, and cigarette smoking were adjusted for the statistical analysis. Relationship between serum lipid levels and genotypes and several environment factors was assessed by multiple linear regression analysis with stepwise modeling. A two-tailed $P$ value less than 0.05 was considered statistically significant.

\section{Results}

3.1. Population Characteristics. The baseline characteristics and serum lipid levels of the Mulao and Han populations are presented in Table 1. The levels of BMI, diastolic blood pressure, and the ratio of ApoAI to ApoB were lower in Mulao than in $\operatorname{Han}(P<0.05)$, whereas the levels of body height, 
LDL-C, ApoB, and the percentages of subjects who consumed alcohol were higher in Mulao than in Han $(P<0.05-0.001)$.

3.2. Results of Electrophoresis and Genotyping. After the genomic DNA of the samples was amplified by PCR and imaged by $2.0 \%$ agarose gel electrophoresis, the purpose gene of $347 \mathrm{bp}$ nucleotide sequences could be found in all samples (Figure 1). The genotypes identified were named according to the presence or absence of the enzyme restriction sites, with a $\mathrm{C}$ to $\mathrm{T}$ transversion at rs4731702 SNP. The presence of the cutting site indicates the $\mathrm{C}$ allele, while its absence indicates the $\mathrm{T}$ allele (cannot be cut). Therefore, the TT genotype is homozygote for the absence of the site (band at $347 \mathrm{bp}$ ), CT genotype is heterozygote for the absence and presence of the site (bands at 347-, 214- and 133-bp), and CC genotype is homozygote for the presence of the site (bands at 214- and 133-bp; Figure 2).

3.3. Results of Sequencing. The results were shown as CC, CT and TT genotypes by PCR-RFLP and the CC, CT, and TT genotypes were also confirmed by sequencing (Figure 3 ), respectively.

3.4. Genotypic and Allelic Frequencies. The genotypic and allelic distribution of the rs4731702 SNP is shown in Table 2. There was no significant difference in either genotypic or allelic frequencies between Mulao and Han. The genotypic and allelic frequencies were different between Han males and females $(P<0.05)$, but not between Mulao males and females. The frequency of minor $\mathrm{T}$ allele in Han was higher in females $(35.8 \%)$ than in males $(30.2 \%, P=0.024)$.

3.5. Genotypes and Serum Lipid Levels. As shown in Table 3, the levels of serum LDL-C and ApoAI in Mulao were different among the three genotypes $(P<0.05)$ after adjusting age, sex, BMI, blood pressure, cigarette smoking, and alcohol consumption; the subjects with TT genotype had higher LDL-C and ApoAI levels than the subjects with CT or CC genotype. For the Han population, the levels of HDL-C and ratio of ApoAI to ApoB were different among the genotypes $(P<0.05)$; the T allele carriers had lower HDL-C levels and the ratio of ApoAI to $A p o B$ than the $T$ allele noncarriers. Subgroup analyses showed that the T allele carriers in Mulao males had higher HDL-C, LDL-C, and ApoAI levels than the T allele noncarriers $(P<0.05)$. The T allele carriers in Han males were associated with lower ApoAI levels and ratio of ApoAI to ApoB than the T allele noncarriers $(P<0.05)$. The subjects with TT genotype in Han females had higher TC, LDL-C, ApoAI, and ApoB levels than the subjects with CT or CC genotype $(P<0.05)$.

3.6. Risk Factors for Serum Lipid Parameters. The correlation between the relative factors and serum lipid parameters in Mulao and Han is depicted in Table 4. Multiple linear regression analyses showed that serum LDL-C levels in Mulao and Han, LDL-C and ApoAI levels in Mulao, and LDL-C levels and the ratio of ApoAI to ApoB in Han were correlated with genotypes $(P<0.05)$, respectively. Serum

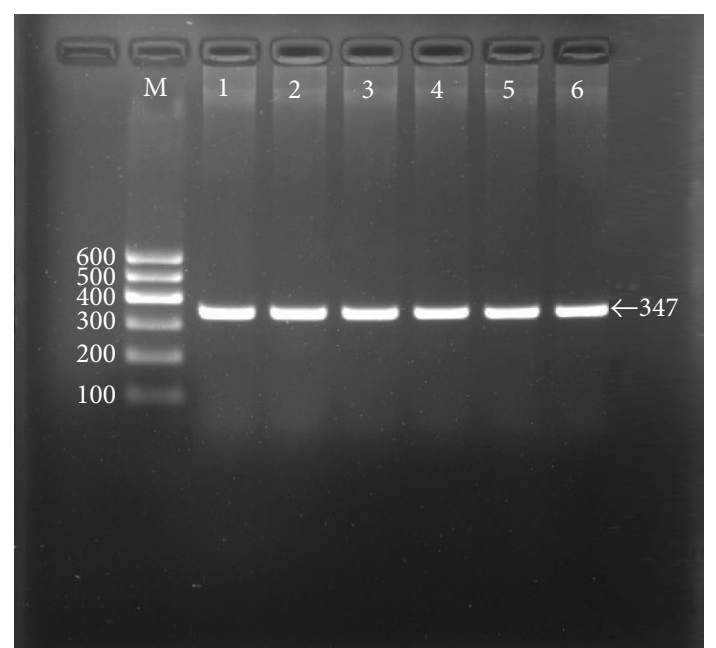

FIGURE 1: Electrophoresis of PCR products of the KLF14 rs4731702 SNP. Lane M, $100 \mathrm{bp}$ marker ladder; lanes 1-6, PCR products (347 bp).

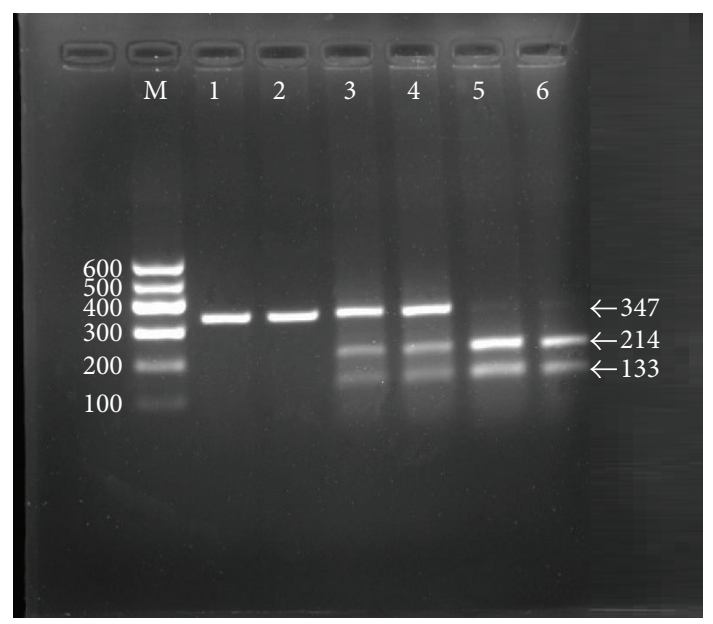

FIGURE 2: Genotyping of the KLF14 rs4731702 SNP. Lane M, $100 \mathrm{bp}$ marker ladder; lanes 1 and 2, TT genotype ( $347 \mathrm{bp}$ ); lanes 3 and 4, CT genotype (347-, 214-, and 133-bp); lanes 5 and 6, CC genotype (214- and 133-bp).

LDL-C and ApoAI levels in Mulao males, HDL-C, ApoAI levels and the ratio of ApoAI to ApoB in Han males, and LDL$\mathrm{C}$ levels in Han females were correlated with genotypes $(P<$ 0.05; Table 5), respectively. Serum lipid parameters were also associated with environmental factors such as age, gender, BMI, waist circumference, blood pressure, blood glucose, cigarette smoking, and alcohol consumption in both ethnic groups $(P<0.05-0.001$; Tables 4 and 5).

\section{Discussion}

Given that genetic factors and interactions with environmental factors are important in common forms of serum lipid levels, prediction of the risk for dyslipidemia on the basis of genetic variants would be beneficial for personalized 
$\uparrow$

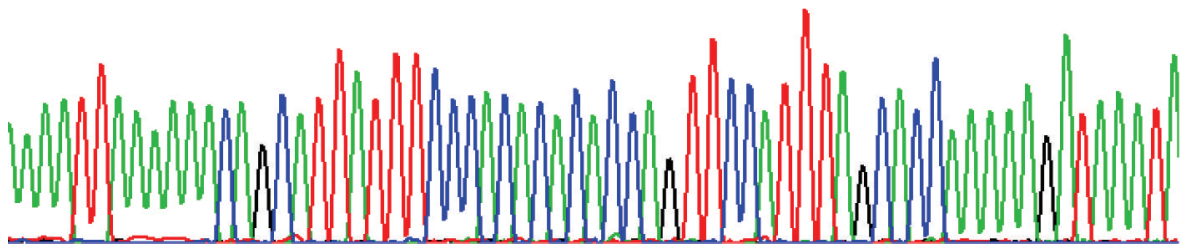

(a)

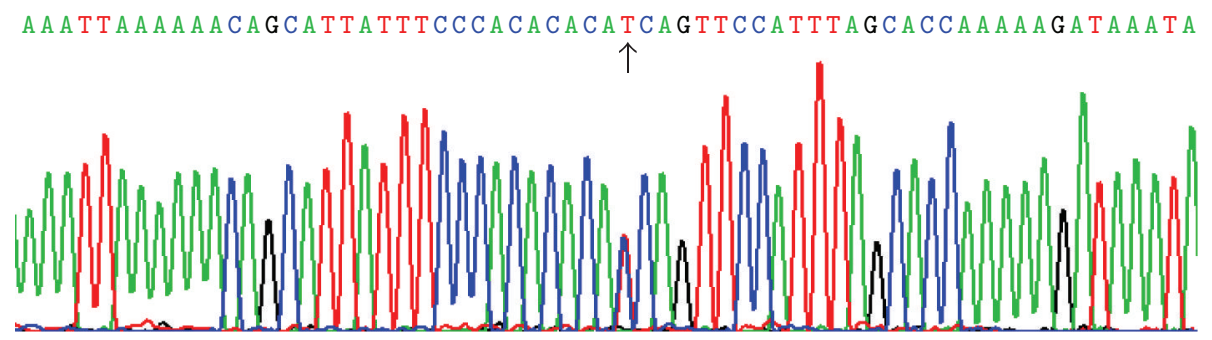

(b)

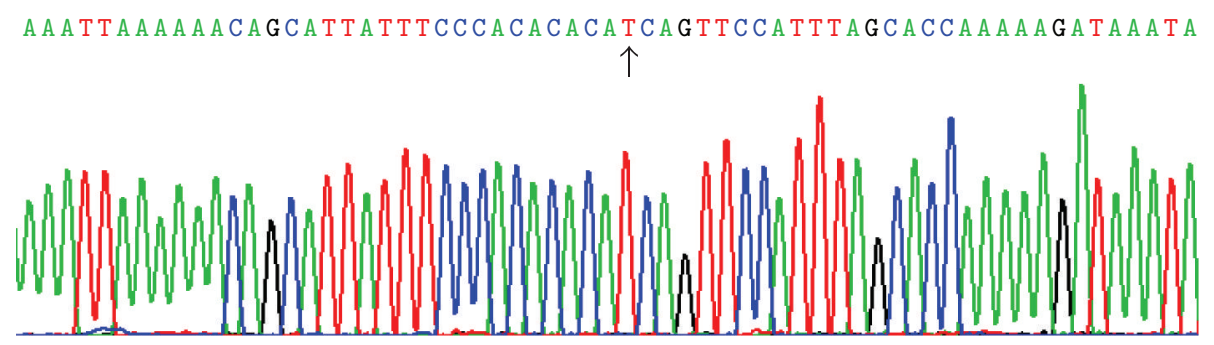

(c)

FIGURE 3: A part of the nucleotide sequence of the KLF14 rs4731702 SNP. (a) CC genotype, (b) CT genotype, and (c) TT genotype.

prevention of this condition [7-9]. Mulao nationality is a relatively conservative and isolated minority in China that retains its regional and special customs. The engagements of Mulao nationality were strictly intraethnic. Traditionally, there was a preference of marriage to relatives of maternal side (mother's brother's daughter) in childhood. Divorce and remarriage were permitted, with little restriction. The twogeneration household is the most common unit of residence. Households are under the control of the father and divided when the sons marry, with only the youngest son remaining with the parents. As a consequence, Mulao population is considered to share the same ethnic ancestry and possess the same genetic background. We believed that some hereditary characteristics and genotypes of lipid metabolism-related genes in this population might be different from those in Han Chinese [35].

The genotypic and allelic frequencies of KLF14 rs4731702 SNP in diverse racial/ethnic groups are not well known. According to the HapMap data, the minor allele frequency of the SNP was $36.7 \%$ in Chinese, $30.0 \%$ in Japanese, $23.3 \%$ in Yoruba, and $45.0 \%$ in European population. Kong et al. [36] demonstrated that the frequency of $\mathrm{T}$ allele was $56.1 \%$ in normal Icelanders. Chen et al. [26] reported that the allelic frequencies of KLF14 rs4731702 SNP were different between atherosclerotic cardiovascular disease and control groups in
Beijing and Taizhou Chinese. The minor T allele frequency in myocardial infarction and ischemic stroke groups was lower than that in control groups. The frequency of T allele was $31.5 \%, 29.1 \%$, and $30.2 \%$ in the three control groups, respectively. In the present study, we showed that the $\mathrm{T}$ allele frequency of KLF14 rs4731702 SNP was 35.7\% in Mulao and $33.4 \%$ in $\operatorname{Han}(P>0.05)$, which was similar to the Beijing and Taizhou Chinese samples [26]. Subgroup analyses showed that the minor allele frequency of rs4731702 SNP in Han was higher in females than in males, and the genotypic distribution was also different between females and males $(P<0.05)$. These results indicated that the prevalence of $\mathrm{T}$ allele of KLF14 rs4731702 SNP may have racial/ethnic as well as gender specificity.

Rare studies have previously reported the direct effect of KLF14 rs4731702 SNP on serum lipid levels. The present study showed a significant association between the rs4731702 SNP and multiple serum lipid parameters in our study populations. The $\mathrm{T}$ allele carriers had higher LDL-C and ApoAI levels in Mulao, while they had lower HDL-C levels and ratio of ApoAI to $A p o B$ in Han than the $T$ allele noncarriers. Moreover, the $\mathrm{T}$ allele carriers were associated with higher HDL-C, LDL-C, and ApoAI levels in Mulao males, lower ApoAI levels and ratio of ApoAI to ApoB in Han males, and higher TC, LDL-C, ApoAI and ApoB levels 
in Han females. The inconsistent association between the two ethnic groups indicated that the correlation of KLF14 rs4731702 SNP and serum lipid levels may have racial/ethnic and/or sex specificity.

KLF is an important part of the regulatory cascade that leads to adipogenesis $[23,24]$. Recently, genetic model of Caenorhabditis elegans was used to elucidate the regulation pathways of $k l f 3$ (a member of KLF family in the nematode) in fat storage. The findings suggested that $k l f 3$ functions as either an activator or a repressor in the regulation of the expression of several genes key to fatty acid $\beta$-oxidation, which resulted in excessive fat deposits and severe fertility defects. This study speculated on the role of worm $k l f 3$ in partial overlapping with that of human KLF14 in fat storage and metabolism [37]. Previous functional studies found that the maternally transmitted T allele of rs4731702 SNP is associated with increased expression of KLF14 in adipose tissue, indicating the presence of a cis expression quantitative trait loci (eQTL) [36]. Small et al. [25] demonstrated that the type 2 diabetes and HDL-C associated cis-acting eQTL of KLF14 acts as a master trans-regulator of adipose gene expression. They detected subcutaneous adipose biopsies from 776 female twins of European ancestry as well as a smaller replication sample. The results suggested a trans-causal link between KLF14 expression and ten genes that were associated with a variety of metabolic syndrome traits including obesity, dyslipidemia, and measures of insulin resistance. Moreover, using large scale genome-wide association study data, they showed that five of the ten genes had nearby SNPs that were associated with key metabolic syndrome traits at genomewide significance. Taken together, the rs4731702 SNP may act in cis to influence the KLF14-associated trans-regulatory network and bring about the cascade of events in lipid metabolism. However, the biological function and detailed role of KLF14 rs4731702 SNP in lipid metabolism need to be further explored.

Here, we also noted that serum lipid parameters were correlated to age, sex, waist circumference, BMI, blood pressure, alcohol consumption, and cigarette smoking in both ethnic groups. These data suggested that the environmental factors also played important roles in determining serum lipid levels. The dietary habits are different between the Mulao and Han populations. Mulao people prefer to eat cold foods along with acidic and spicy dishes, local bean soy sauce, pickled vegetables, and animal offals which contain abundant saturated fatty acids. Over the past several decades evidence has accumulated suggesting that dietary intake of saturated and trans-fat raises blood cholesterol concentrations and CVD risk [3841]. A meta-analysis demonstrated that dietary interventions significantly decreased plasma lipids and lipoproteins, and for every $1 \%$ decrease in energy consumed as dietary saturated fatty acid, TC decreased by $0.056 \mathrm{mmol} / \mathrm{L}$ and LDLC by $0.05 \mathrm{mmol} / \mathrm{L}$. Furthermore, for every 1-kg decrease in body weight, TG decreased by $0.011 \mathrm{mmol} / \mathrm{L}$ and HDL$\mathrm{C}$ increased by $0.011 \mathrm{mmol} / \mathrm{L}$ [42]. In addition, numerous studies reported that portfolio diets replace saturated fatty acids with polyunsaturated fatty acids, monounsaturated fatty acids, carbohydrates, and mixed sources and partial substitution of protein would improve serum lipid levels and be beneficial in prevention of CVD $[43,44]$. We also found that the percentages of subjects who consumed alcohol were higher in Mulao than in Han nationalities $(P<0.05)$. Many studies showed that moderate alcohol intake has been associated with reduced cardiovascular events [45-47]. The beneficial effects of alcohol on CVD have been ascribed to the increase in HDL-C and ApoAI levels [48]. However, alcohol can be addictive, and high intake can be associated with serious adverse health including hypertriglyceridemia, hypertension, and liver damage. Like any other source of carbohydrates, alcohol can increase plasma TG levels and can serve as a source of excess calories [49]. It was reported that the alcohol intake of $60 \mathrm{~g} /$ day increases the TG levels by about $0.19 \mathrm{mg} / \mathrm{dL}$ per 1 gram of alcohol consumed [50]. Onat et al. [51] also showed that alcohol consumption was positively associated with TG, LDL-C, and ApoB in men and negatively correlated with TG and/or not correlated with LDL-C and ApoB in women. Nevertheless, another research indicated that the effects of alcohol consumption on LDL-C appear to vary by specific patient types or patterns of alcohol intake, and sex as well as genetic variants [52]. Consequently, the joint effects of different dietary habits, lifestyles, and environmental factors probably further modify the association of genetic variations and serum lipid levels in our study populations.

There are several potential limitations in our study. First, we were not able to alleviate the effect of diet during the statistical analysis since the diet intake was self-reported and difficult to classify. Second, we only measured serum TC, TG, HDL-C, LDL-C, ApoAI, ApoB levels, and the ratio of ApoAI to ApoB and detected their associations with rs4731702 SNP without comprehensive measurements of the subclasses lipoproteins such as HDL2, HDL3, small dense LDL, and large buoyant LDL. However, serum TC, HDL-C, and LDL$\mathrm{C}$ are the most important indicators for dyslipidemia and are also the phenotypes of clinical routine testing. We believed that the SNP associated with these lipid parameters may add predictive information for the development of dyslipidemia and CVD. Third, although we observe significant association of rs4731702 SNP and serum lipid levels, there are still many unmeasured environmental and genetic factors that needed to be considered. The interactions of gene-gene, gene-environment, and environment-environment on serum lipid levels are remained to be determined. Moreover, we recognize the limited power to provide a more significant advance in understanding the full impact of rs4731702 SNP on lipoprotein metabolism. The association of the rs 4731702 SNP, KLF14 expression in adipose tissue and plasma lipid levels should be detected in further investigations.

\section{Conclusions}

The present study shows that genotypic and allelic frequencies of KLF14 rs4731702 SNP were not different between the Mulao and Han populations, whereas difference in the genotypic and allelic frequencies of KLF14 rs4731702 SNP was observed between Han males and females. The association of KLF14 rs4731702 SNP and serum lipid levels is 
different between the two ethnic groups. These trends of association suggest that this SNP might have racial/ethnic or gender specificity. The differences in the association of KLF14 rs4731702 SNP and serum lipid levels between the two ethnic groups might partly result from the differences in gene-environmental interactions.

\section{Acknowledgment}

This study was supported by the National Natural Science Foundation of China (no. 30960130).

\section{References}

[1] R. Lozano, M. Naghavi, K. Foreman et al., "Global and regional mortality from 235 causes of death for 20 age groups in 1990 and 2010: a systematic analysis for the global burden of disease study 2010," The Lancet, vol. 380, no. 9859, pp. 2095-2128, 2012.

[2] K. G. M. M. Alberti, R. H. Eckel, S. M. Grundy et al., "Harmonizing the metabolic syndrome: a joint interim statement of the international diabetes federation task force on epidemiology and prevention; national heart, lung, and blood institute; American heart association; world heart federation; international atherosclerosis society; and international association for the study of obesity," Circulation, vol. 120, no. 16, pp. 1640-1645, 2009.

[3] R. Clarke, J. R. Emberson, S. Parish et al., "Cholesterol fractions and apolipoproteins as risk factors for heart disease mortality in older men," Archives of Internal Medicine, vol. 167, no. 13, pp. 1373-1378, 2007.

[4] K. Kuulasmaa, H. Tunstall-Pedoe, A. Dobson et al., "Estimation of contribution of changes in classic risk factors to trends in coronary-event rates across the WHO MONICA project populations," The Lancet, vol. 355, no. 9205, pp. 675-687, 2000.

[5] M. R. Law, N. J. Wald, and A. R. Rudnicka, "Quantifying effect of statins on low density lipoprotein cholesterol, ischaemic heart disease, and stroke: systematic review and meta-analysis," British Medical Journal, vol. 326, no. 7404, pp. 1423-1427, 2003.

[6] R. Jackson, C. M. M. Lawes, D. A. Bennett, R. J. Milne, and A. Rodgers, "Treatment with drugs to lower blood pressure and blood cholesterol based on an individual's absolute cardiovascular risk," The Lancet, vol. 365, no. 9457, pp. 434-441, 2005.

[7] S. Kathiresan, C. J. Willer, G. M. Peloso et al., "Common variants at 30 loci contribute to polygenic dyslipidemia," Nature Genetics, vol. 41, no. 1, pp. 56-65, 2009.

[8] Y. Ruixing, F. Qiming, Y. Dezhai et al., "Comparison of demography, diet, lifestyle, and serum lipid levels between the Guangxi Bai Ku Yao and Han populations," Journal of Lipid Research, vol. 48, no. 12, pp. 2673-2681, 2007.

[9] Y. Ruixing, C. Yuming, P. Shangling et al., "Effects of demographic, dietary and other lifestyle factors on the prevalence of hyperlipidemia in Guangxi Hei Yi Zhuang and Han populations," European Journal of Cardiovascular Prevention and Rehabilitation, vol. 13, no. 6, pp. 977-984, 2006.

[10] L. Perusse, T. Rice, J. P. Despres et al., "Familial resemblance of plasma lipids, lipoproteins and postheparin lipoprotein and hepatic lipases in the HERITAGE Family study," Arteriosclerosis, Thrombosis, and Vascular Biology, vol. 17, no. 11, pp. 3263-3269, 1997.

[11] D. A. Heller, U. de Faire, N. L. Pedersen, G. Dahlen, and G. E. McClearn, "Genetic and environmental influences on serum lipid levels in twins," The New England Journal of Medicine, vol. 328, no. 16, pp. 1150-1156, 1993.

[12] L. Dumitrescu, C. L. Carty, K. Taylor et al., "Genetic determinants of lipid traits in diverse populations from the population architecture using genomics and epidemiology (PAGE) study," PLoS Genetics, vol. 7, no. 6, Article ID e1002138, 2011.

[13] T. M. Teslovich, K. Musunuru, A. V. Smith et al., "Biological, clinical and population relevance of 95 loci for blood lipids," Nature, vol. 466, no. 7307, pp. 707-713, 2010.

[14] D. I. Chasman, G. Paré, S. Mora et al., "Forty-three loci associated with plasma lipoprotein size, concentration, and cholesterol content in genome-wide analysis," PLoS Genetics, vol. 5, no. 11, Article ID e1000730, 2009.

[15] P. Jeemon, K. Pettigrew, C. Sainsbury, D. Prabhakaran, and S. Padmanabhan, "Implications of discoveries from genomewide association studies in current cardiovascular practice," The World Journal of Cardiology, vol. 3, no. 7, pp. 230-247, 2011.

[16] T. Ohshige, M. Iwata, S. Omori et al., "Association of new loci identified in European genome-wide association studies with susceptibility to type 2 diabetes in the Japanese," PLOS ONE, vol. 6, no. 10, Article ID e26911, 2011.

[17] B. F. Voight, L. J. Scott, V. Steinthorsdottir et al., "Twelve type 2 diabetes susceptibility loci identified through large-scale association analysis," Nature Genetics, vol. 42, no. 7, pp. 579-589, 2010.

[18] S. D. Rees, M. Z. I. Hydrie, A. S. Shera et al., "Replication of 13 genome-wide association (GWA)-validated risk variants for type 2 diabetes in Pakistani populations," Diabetologia, vol. 54, no. 6, pp. 1368-1374, 2011.

[19] L. Parker-Katiraee, A. R. Carson, T. Yamada et al., "Identification of the imprinted KLF14 transcription factor undergoing human-specific accelerated evolution," PLoS genetics, vol. 3, no. 5, article e65, 2007.

[20] D. T. Dang, J. Pevsner, and V. W. Yang, "The biology of the mammalian Krüppel-like family of transcription factors," International Journal of Biochemistry and Cell Biology, vol. 32, no. 11-12, pp. 1103-1121, 2000.

[21] R. Pearson, J. Fleetwood, S. Eaton, M. Crossley, and S. Bao, "Krüppel-like transcription factors: a functional family," International Journal of Biochemistry and Cell Biology, vol. 40, no. 10, pp. 1996-2001, 2008.

[22] J. van Vliet, L. A. Crofts, K. G. R. Quinlan, R. Czolij, A. C. Perkins, and M. Crossley, "Human KLF17 is a new member of the Sp/KLF family of transcription factors," Genomics, vol. 87, no. 4, pp. 474-482, 2006.

[23] C. W. Brey, M. P. Nelder, T. Hailemariam, R. Gaugler, and S. Hashmi, "Krüppel-like family of transcription factors: an emerging new frontier in fat biology," International Journal of Biological Sciences, vol. 5, no. 6, pp. 622-636, 2009.

[24] B. B. McConnell and V. W. Yang, "Mammalian Krüppel-like factors in health and diseases," Physiological Reviews, vol. 90, no. 4, pp. 1337-1381, 2010.

[25] K. S. Small, Å. K. Hedman, E. Grundberg et al., "Identification of an imprinted master trans regulator at the KLF14 locus related to multiple metabolic phenotypes," Nature Genetics, vol. 43, no. 6, pp. 561-564, 2011.

[26] X. Chen, S. Li, Y. Yang et al., "Genome-wide association study validation identifies novel loci for atherosclerotic cardiovascular disease," Journal of Thrombosis and Haemostasis, vol. 10, no. 8, pp. 1508-1514, 2012. 
[27] L. Xu, Q.-Y. Deng, S.-F. Li, L.-N. Zhou, J.-C. Gong, and B.Y. Wei, "Genetic analysis of Mulao nationality using 15 short tandem repeats," Chinese Journal of Medical Genetics, vol. 25, no. 1, pp. 96-100, 2008.

[28] Q. Li, R.-X. Yin, T.-T. Yan et al., "Association of the GALNT2 gene polymorphisms and several environmental factors with serum lipid levels in the Mulao and Han populations," Lipids in Health and Disease, vol. 10, article 160, 2011.

[29] T.-T. Yan, R.-X. Yin, Q. Li et al., "Sex-specific association of rs16996148 SNP in the NCAN/CILP2/PBX4 and serum lipid levels in the Mulao and Han populations," Lipids in Health and Disease, vol. 10, article 248, 2011.

[30] S. Irving, "An epidemiological study of cardiovascular and cardiopulmonary disease risk factors in four populations in the People's Republic of China: baseline report from the P.R.C.U.S.A. collaborative study," Circulation, vol. 85, no. 3, pp. 10831096, 1992.

[31] L. Aung, R.-X. Yin, D.-F. Wu et al., "Association of the TRIB1 tribbles homolog 1 gene rs17321515 A>G polymorphism and serum lipid levels in the Mulao and Han populations," Lipids in Health and Disease, vol. 10, article 230, 2011.

[32] D.-F. Wu, R.-X. Yin, L. H. H. Aung et al., "Sex-specific association of ACAT-1 rs1044925 SNP and serum lipid levels in the hypercholesterolemic subjects," Lipids in Health and Disease, vol. 11, article 9, 2012.

[33] Y. Ruixing, P. Shangling, C. Hong et al., "Diet, alcohol consumption, and serum lipid levels of the middle-aged and elderly in the Guangxi Bai Ku Yao and Han populations," Alcohol, vol. 42, no. 3, pp. 219-229, 2008.

[34] B.-F. Zhou, "Predictive values of body mass index and waist circumference for risk factors of certain related diseases in Chinese adults-study on optimal cut-off points of body mass index and waist circumference in Chinese adults," Biomedical and Environmental Sciences, vol. 15, no. 1, pp. 83-96, 2002.

[35] D. F. Wu, R. X. Yin, T. T. Yan et al., "The SCARB1 rs5888 SNP and serum lipid levels in the Guangxi Mulao and Han populations," International Journal of Medical Sciences, vol. 9, no. 8, pp. 715724, 2012.

[36] A. Kong, V. Steinthorsdottir, G. Masson et al., "Parental origin of sequence variants associated with complex diseases," Nature, vol. 462, no. 7275, pp. 868-874, 2009.

[37] J. Zhang, R. Bakheet, R. S. Parhar et al., "Regulation of fat storage and reproduction by Krüppel-like transcription factor KLF3 and fat-associated genes in Caenorhabditis elegans," Journal of Molecular Biology, vol. 411, no. 3, pp. 537-553, 2011.

[38] M. R. Garcia-Palmieri, J. Tillotson, E. Cordero et al., "Nutrient intake and serum lipids in urban and rural Puerto Rican men," The American Journal of Clinical Nutrition, vol. 30, no. 12, pp. 2092-2100, 1977.

[39] O. I. Bermudez, W. Velez-Carrasco, E. J. Schaefer, and K. L. Tucker, "Dietary and plasma lipid, lipoprotein, and apolipoprotein profiles among elderly Hispanics and non-Hispanics and their association with diabetes," The American Journal of Clinical Nutrition, vol. 76, no. 6, pp. 1214-1221, 2002.

[40] R. Solá, M. Fitó, R. Estruch et al., "Effect of a traditional Mediterranean diet on apolipoproteins B, A-I, and their ratio: a randomized, controlled trial," Atherosclerosis, vol. 218, no. 1, pp. 174-180, 2011.

[41] E. A. Valente, M. E. Sheehy, J. J. Avila, J. A. Gutierres, M. J. Delmonico, and I. E. Lofgren, "The effect of the addition of resistance training to a dietary education intervention on apolipoproteins and diet quality in overweight and obese older adults," Journal of Clinical Interventions in Aging, vol. 6, no. 1, pp. 235-241, 2011.

[42] S. Yu-Poth, G. Zhao, T. Etherton, M. Naglak, S. Jonnalagadda, and P. M. Kris-Etherton, "Effects of the National Cholesterol Education Program's Step I and Step II dietary intervention programs on cardiovascular disease risk factors: a meta-analysis," The American Journal of Clinical Nutrition, vol. 69, no. 4, pp. 632-646, 1999.

[43] L. J. Appel, F. M. Sacks, V. J. Carey et al., "Effects of protein, monounsaturated fat, and carbohydrate intake on blood pressure and serum lipids: results of the OmniHeart randomized trial," The Journal of the American Medical Association, vol. 294, no. 19, pp. 2455-2464, 2005.

[44] K. Musunuru, "Atherogenic dyslipidemia: cardiovascular risk and dietary intervention," Lipids, vol. 45, no. 10, pp. 907-914, 2010.

[45] R. Doll, R. Peto, E. Hall, K. Wheatley, and R. Gray, "Mortality in relation to consumption of alcohol: 13 years' observations on male British doctors," British Medical Journal, vol. 309, no. 6959, pp. 911-918, 1994.

[46] C. A. Camargo Jr., C. H. Hennekens, J. M. Gaziano, R. J. Glynn, J. E. Manson, and M. J. Stampfer, "Prospective study of moderate alcohol consumption and mortality in US male physicians," Archives of Internal Medicine, vol. 157, no. 1, pp. 79-85, 1997.

[47] M. Flesch, S. Rosenkranz, E. Erdmann, and M. Böhm, "Alcohol and the risk of myocardial infarction," Basic Research in Cardiology, vol. 96, no. 2, pp. 128-135, 2001.

[48] E. R. de Oliveira e Silva, D. Foster, M. M. Harper et al., "Alcohol consumption raises HDL cholesterol levels by increasing the transport rate of apolipoproteins A-I and A-II," Circulation, vol. 102, no. 19, pp. 2347-2352, 2000.

[49] I. J. Goldberg, L. Mosca, M. R. Piano, and E. A. Fisher, "Wine and your heart: a science advisory for healthcare professionals from the nutrition committee, council on epidemiology and prevention, and council on cardiovascular nursing of the American Heart Association," Circulation, vol. 103, no. 3, pp. 472-475, 2001.

[50] M. J. Stampfer, R. M. Krauss, J. Ma et al., "A prospective study of triglyceride level, low-density lipoprotein particle diameter, and risk of myocardial infarction," The Journal of the American Medical Association, vol. 276, no. 11, pp. 882-888, 1996.

[51] A. Onat, G. Hergenc, D. Dursunoglu et al., "Associations of alcohol consumption with blood pressure, lipoproteins, and subclinical inflammation among Turks," Alcohol, vol. 42, no. 7, pp. 593-601, 2008.

[52] E. A. Brinton, "Effects of ethanol intake on lipoproteins and atherosclerosis," Current Opinion in Lipidology, vol. 21, no. 4, pp. 346-351, 2010. 


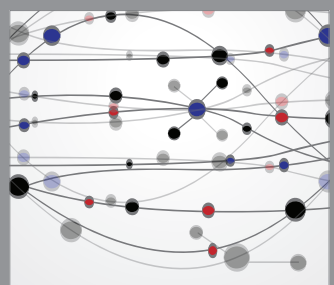

The Scientific World Journal
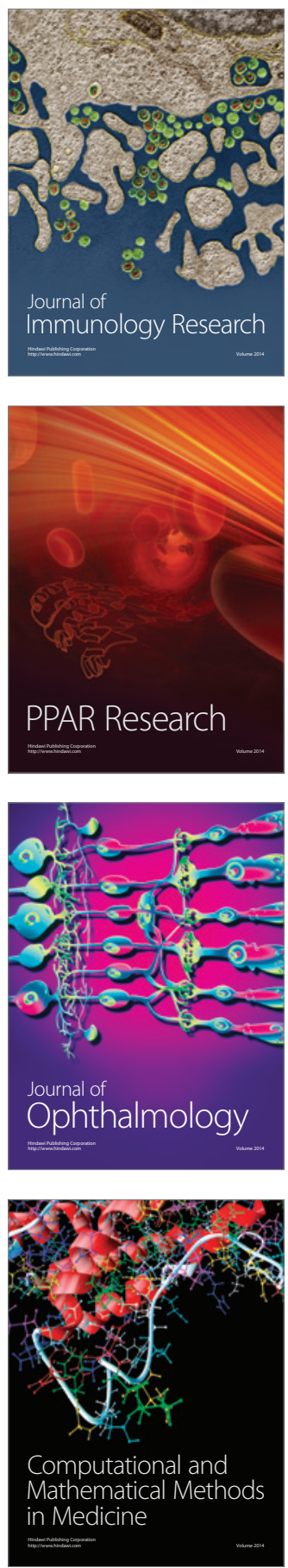

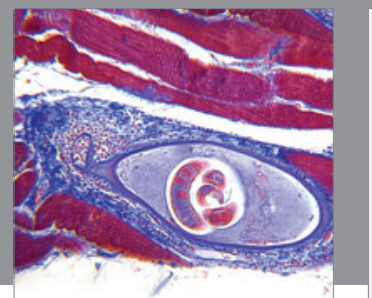

Gastroenterology

Research and Practice
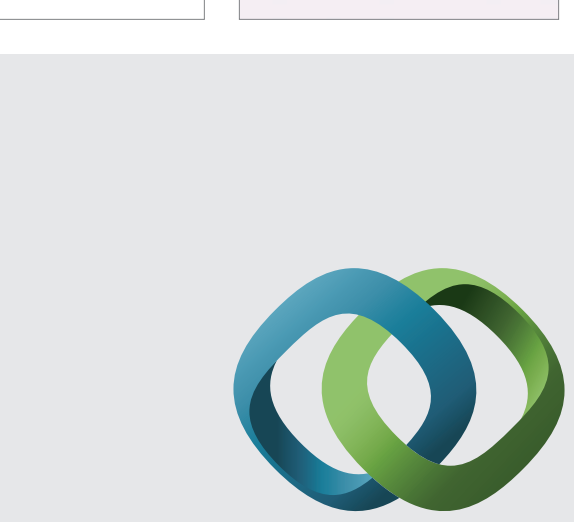

\section{Hindawi}

Submit your manuscripts at

http://www.hindawi.com
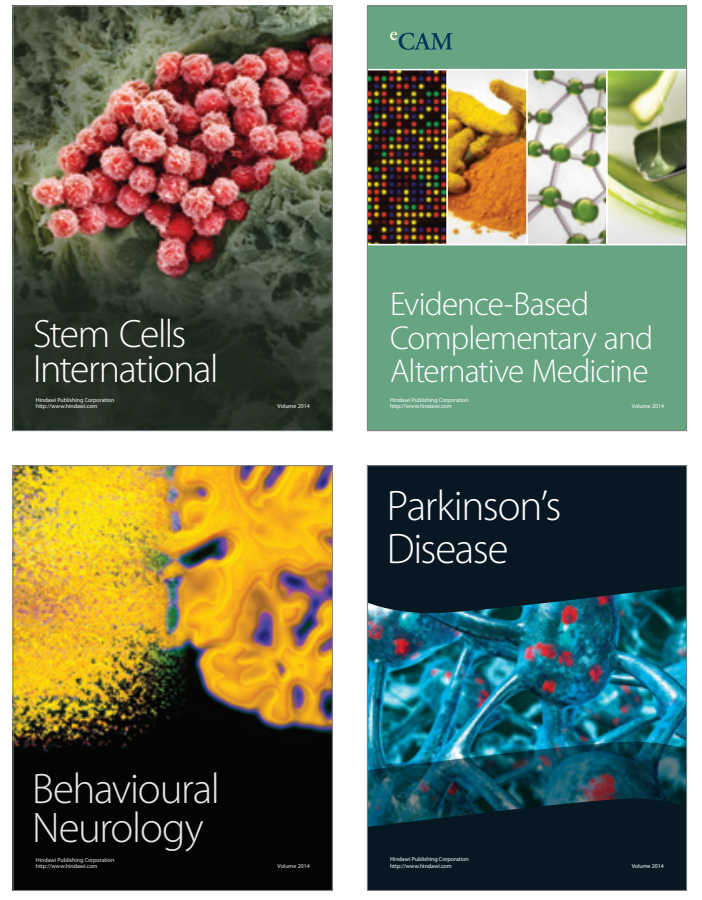
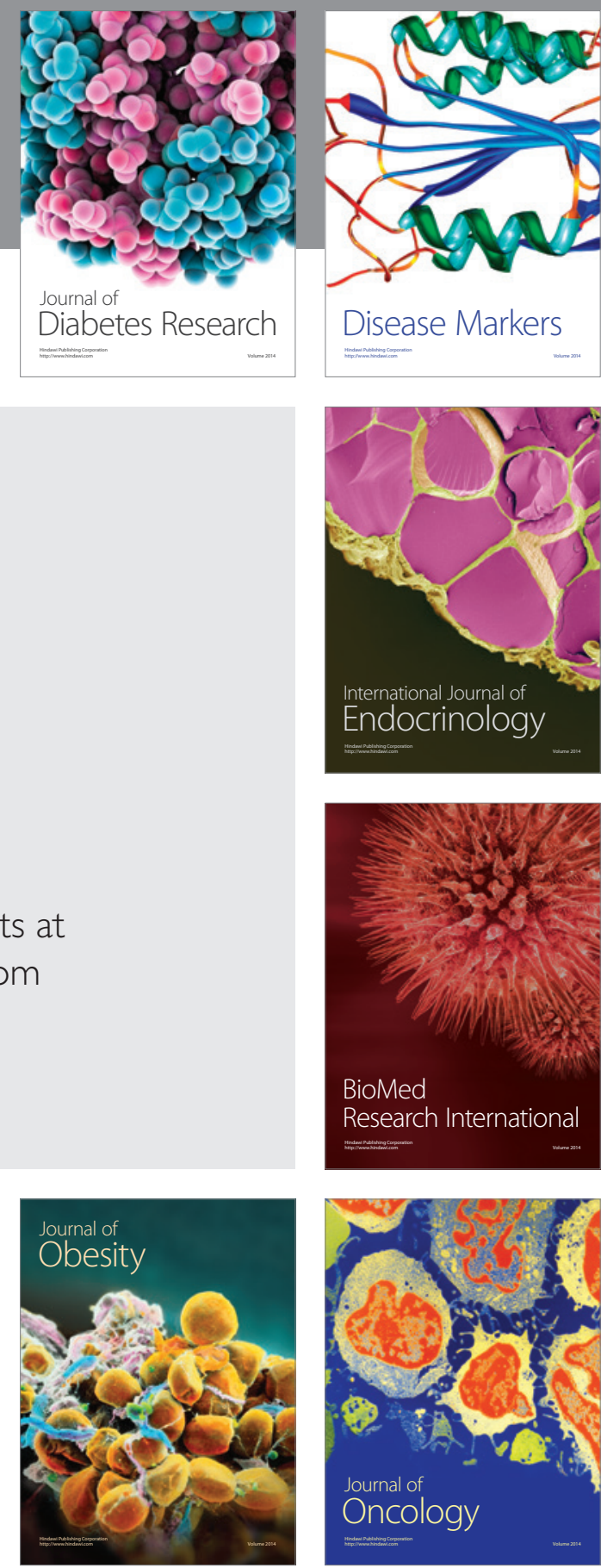

Disease Markers
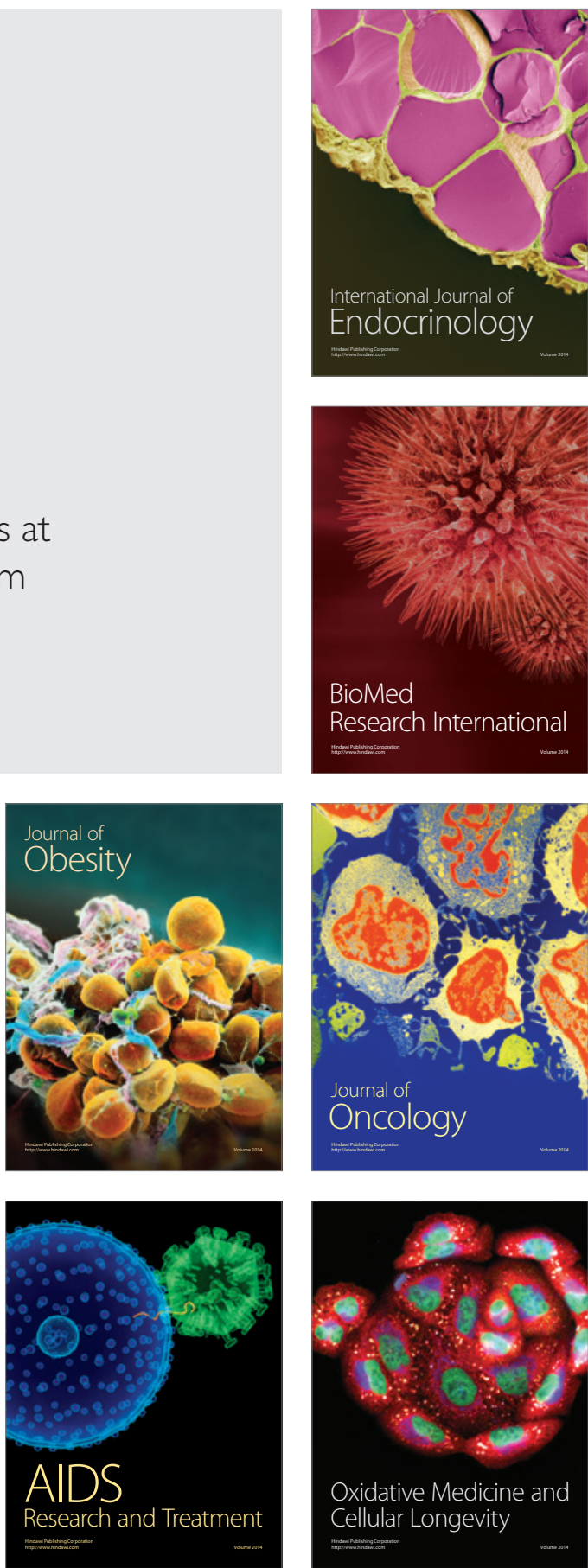